\title{
STM Observation of the Si(111) - (7×7) Reconstructed Surface Modified by Excess Phosphorus Doping
}

\author{
Hirulak D. Siriwardena ${ }^{1}$, Toru Yamashita ${ }^{2}$, Masaru Shimomura ${ }^{3}$ \\ ${ }^{1,3}$ Graduate School of Science and Technology, Shizuoka University, Japan \\ ${ }^{2,3}$ Graduate School of Integrated Science and Technology, Shizuoka University, Japan
}

\section{Article Info \\ Article history: \\ Received Aug 23, 2017 \\ Revised Nov 12, 2017 \\ Accepted Nov 26, 2017}

Keyword:

Excess phosphorous doping $\operatorname{Si}(111)-(7 \times 7)$

STM

STS

\begin{abstract}
The electronic properties of semiconductor surfaces change readily upon changing the carrier densities by controlling the dopant concentration. Additionally, excess dopant atoms can exert electric field which would affect the molecular adsorption process and could be used to manipulate the dynamic movement of confined molecules. A mechanism can be developed to control the molecular dynamic movement on modified semiconductor surface by dopants thus changing the effect of the electric field on the active molecules. In this study, the Si(111) surface was doped with phosphorus excessively using thermal diffusion process. The surface was then reconstructed to the $7 \times 7$ configuration via heating under UHV conditions and then studied through STM and STS techniques. The protrusions due to surface and subsurface $\mathrm{P}$ atoms appear brighter due to the lone electron pair. The $7 \times 7$ reconstruction would be destabilized after a critical $P$ substitution of $\mathrm{Si}$-adatom concentration due to high surface strain result in P-terminated $(6 \sqrt{3} \times 6 \sqrt{3}) R 30^{\circ}$ reconstruction.
\end{abstract}

Copyright $(0) 2017$ Institute of Advanced Engineering and Science. All rights reserved.

\section{Corresponding Author:}

Masaru Shimomura,

Graduate School of Science and Technology,

Shizuoka University,

3-5-1 Johoku, Nakaku, Hamamatsu 432-8011, Japan.

E-mail: shimomura.masaru@shizuoka.ac.jp

\section{INTRODUCTION}

Dopant atoms are of extreme importance in semiconductor physics and applications since the dopant atoms determine the electronic properties [1]. The mostly affected electrical property component is the charge carrier concentration of the material. The electron and holes concentration of a semiconductor under thermal equilibrium is equal to each other. Introducing dopant atoms increase the conductivity of the substrate due to the increment in carrier concentrations. Very highly doped or degenerate semiconductor materials have conductivity property levels equivalent to metals and therefore show different properties when compared with typical semiconductors [2]. There are two basic methods which an impurity dopant atom may penetrate into a lattice. The dopant atom can substitute an atom of the host crystal which is known as the substitutional impurity. Otherwise, the dopant can remain in a position between the lattice site, which is known as the interstitial impurity. The best and well-recognized impurities are the atoms from group III and $\mathrm{V}$ of the periodic table for the group IV semiconductors. These atoms can enter the host lattice and can substitute by forming covalent bonds [3]. When phosphorus (P) atom enters the Si semiconductor, it occupied one of the lattice sites in the Si crystal. To form four stable bindings among the Si host atoms, $\mathrm{P}$ has to lose one electron, which is an element having five outer shell electrons, so four electrons can implement into the covalent structure. The impurity $\mathrm{P}$ atom becomes a positively charged ion after losing the election in the lattices and thus starts to exert a perturbing force on its nearby host atoms [2]. The perturbing potential acts similar to a Coulomb potential as a proton is placed at the impurity site. 
Several studies on molecules confined in self-assembled molecular corral structures, as shown in the STM image of Figure 1(a), have already been carried out [4], [5]. It has observed that the trimethylphosphine (TMP) molecules adsorbed on a corner-adatom position of $\mathrm{Si}(111)$ - $(7 \times 7)$ reconstructed surface move dynamically inside self-assembled pyrrole molecular fences due to the mutual repulsive force exerted by the TMP adsorbates [5], [6]. Further studies on the molecular system confirm that this dynamic movement also affected by various tunneling electron injection conditions, especially sample bias voltage because of the influence of the antibonding orbitals of the $\mathrm{Si}-\mathrm{P}$ bond [7]. We suggest that an electric field can be formed if there is an excess amount of positive ions due to high $\mathrm{P}$ doing and this electric field can affect the dynamic movement of adsorbed molecules on the substrate surface. If the pyrrole and TMP molecular structure can be placed in between a nanogap and if the dynamic movement of TMP is controlled, a molecular level switch like device can be fabricated.
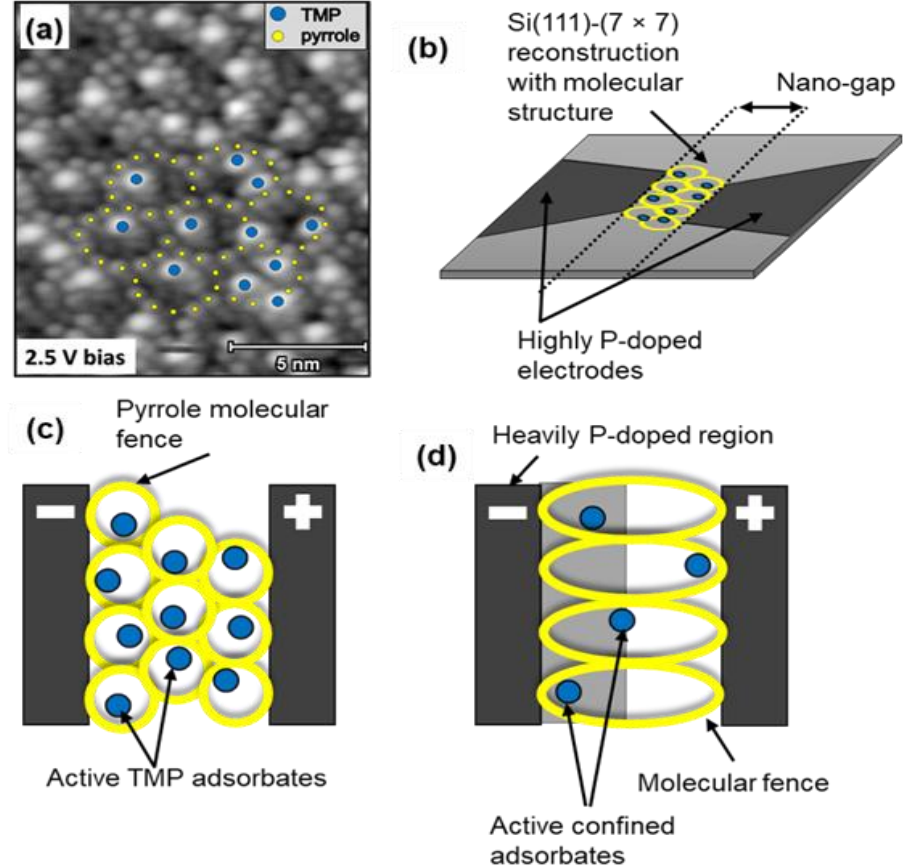

Figure 1. (a) STM image of confined TMP adsorbates in self-assembled pyrrole molecular corrals on $\mathrm{Si}(111)$ - (7×7) reconstructed surface, (b) Schematic illustration of the purposed single molecular electronic device. Top view of the device, (c) TMP adsorbates confined in pyrrole molecular fences fabricated in the nano-gap and (d) TMP adsorbates confined in extended pyrrole molecular fences fabricated on heavily

P-doped area between the nano-gap

For this process, a nano-gap electrode with a spacing about $50 \mathrm{~nm}$ has to be fabricated on the highly P-doped Si (111)-(7×7) reconstructed surface as illustrated in Figure 1(b). Then the confined TMP molecular arrangement is fabricated via self-assembly process. Since the dynamic behavior of TMP adsorbates under different sample bias conditions is known, it is possible to precisely manipulate the movement of the TMP adsorbates with respect to the applied voltage. It is expected that the dynamically active TMP adsorbates move towards the direction of the cathode of the nanogap since TMP has a positive charge around it, as shown in Figure 1(c). The molecular arrangement can act as a storage device or a molecular switch, as mentioned earlier if the movement is reversible. Figure 1(d) depicts the schematic of proposed molecularlevel device with an extended molecular fence. It is expected that the P-doping would affect the electron transfer between the nanogap and therefore, it is important to study the effects of doping as discussed in this paper.

The elemental semiconductor surfaces, such as $\mathrm{Si}$, are terminated by a bulk lattice plane, and any dangling bond usually should hold one electron [8]. If $\mathrm{P}$ substitutes surface adatom sites, dangling bonds would contain lone electron pair which would affect heavily on charge transfer process which occurs during molecular adsorption. Therefore, the P doping was carried out by the thermal diffusion process, and the STM 
investigation of the highly P-doped $\mathrm{Si}(111)-(7 \times 7)$ surface was performed. Also, the surface was further analyzed by STS measurements to determine the electronic properties after the doping process.

\section{RESEARCH METHOD}

Diffusion doping method was adapted in order to prepare $\mathrm{Si}(111)$ substrates with high $\mathrm{P}$ dopant concentrations. It is important to define the three prominent factors that determine the dopant concentration: i.e. diffusion temperature, diffusion time and the oxide mask thickness [9]. In this study n-type (P-doped) $\mathrm{Si}(111)$ samples with the dimension of $7 \times 7 \mathrm{~mm}^{2}$ were first cleaned by wet etching using $\mathrm{HF}$ :deionized water $=1: 20$ solution and piranha solution $\left(\mathrm{H}_{2} \mathrm{SO}_{4}: \mathrm{H}_{2} \mathrm{O}_{2}=4: 1\right)$. A thin oxide layer was deposited on the Si surfaces after the HF wet etching process using dry oxidation furnace. The process was performed at $650^{\circ} \mathrm{C}$ for 10 minutes under a constant $\mathrm{O}_{2}$ gas flow. The surface of the Si wafer gets oxidized when exposed to oxygen and form a $\mathrm{SiO}_{2}$ layer. Then the P source, OCD P-59230 was spin coated on the samples at 3000RPM for 15 seconds. Samples were then introduced into the open-furnace-tube diffusion system and pre-backing process was carried out under constant $\mathrm{N}_{2}$ gas flow for 30 minutes at $600^{\circ} \mathrm{C}$. After that, the 'drive in' process is carried out under constant $\mathrm{N}_{2}$ gas flow for 10 minutes at a diffusion temperature of $950^{\circ} \mathrm{C}$. After the doping process, the protective oxide layer and remaining OCD layer was etched out using the wet etching process. Then the sheet resistance measurements were carried out using the four-probe (K750RS Kyowariken) measurement technique. Secondary ion mass spectrometric (SIMS, AXIS-ULTRA DLD) measurements were further performed on the P-doped Si substrates in order to confirm the doping has taken place. The highly P-doped Si (111) substrates were then cut into $1 \mathrm{~mm} \times 7 \mathrm{~mm}$ pieces and introduced in to the main chamber of the STM (JEOL JSTM 4500XT) after the cleaning process. The $(7 \times 7)$ reconstruction was achieved by heating the sample up to $\sim 1100^{\circ} \mathrm{C}$, maintaining the UHV conditions. However, it is suitable to limit the number of flashing cycles since the 'drive in' of surface $\mathrm{P}$ can occur and surface properties will not be modified significantly if $\mathrm{P}$ dopant go deep into the Si lattice.

\section{RESULTS AND ANALYSIS}

During the four-probe-point measurement, the four probes are brought into contain with the sample surface and the voltage drop $(V)$ is measured between the inner two probes when a current $(I)$ is passed through the outer two probes and sheet resistance $\left(R_{s}\right)$ can be measured directly by using $\Omega / \square$ (ohms per square) mode. Additionally, the $R_{s}$ value can be calculated by using $R_{S}=\frac{V}{I} C F$ equation if value $V / I$ is determined from the four-probe-point system where, $C F$ is the correction factor which depends on the probe arrangement and the sample dimension. The value of the $C F$ is equal to 4.53 when the sample width is significantly larger than the probe spacing [10]. The P-doping of the samples were confirmed by the measured $R_{s}$ values and tabulated in Table 1. An average $R_{s}$ value of $23.923 \Omega / \square$ was observed for the undoped sample and the average $R_{S}$ value get reduced to $15.760 \Omega / \square$ after the P-doping process was performed.

Table 1. The four-probe point measurements recorded before and after $\mathrm{P}$ doping process

\begin{tabular}{lcccccc}
\hline \multicolumn{9}{c}{ Before doping } & \multicolumn{2}{c}{ After doping } \\
\hline & $R_{s}(\Omega / \square)$ & $V / I(\Omega)$ & $R_{s}(\Omega / \square)=\frac{V}{I} 4.53$ & $R_{s}(\Omega / \square)$ & $V / I(\Omega)$ & $R_{s}(\Omega / \square)=\frac{V}{I} 4.53$ \\
Sample & 23.170 & 5.118 & & 15.830 & 3.4840 & \\
& 23.839 & 5.253 & & 15.470 & 3.4052 & \\
Average & 24.689 & 5.471 & & 15.450 & 3.4204 & 15.760 \\
\hline
\end{tabular}

For SIMS measurements, $\mathrm{Ar}^{+}$cluster ion beam was used for the etching process and secondary ion intensity of $\mathrm{Si}, \mathrm{P}$, and $\mathrm{O}$ was detected while the etching process is carried out for both doped and undoped samples against time. Figure 2 shows the raw SIMS data obtained for doped and undoped Si(111) samples.

The raw data was further analyzed by plotting $\mathrm{P} / \mathrm{Si}$ atom ratio for the doped and undoped samples and is shown in Figure 2(c). From the plot, it can be concluded that the sample is doped with P significantly.

Figure 3 shows filled state constant current images of P-doped substrate surfaces. Randomly spread bright protrusions can be observed in Figure 3(a) which shows a large area of the surface. Also, it is noted that surface defects in $(7 \times 7)$ surface are higher than the number of defects normally observed on a clean (7×7) reconstruction. The bright protrusion in Figure 3(b) provides convincing evidence that the surface $\mathrm{Si}$ corner and center-adatoms have been replaced by the $\mathrm{P}$ dopants. High contrast in the filled state image 
corresponds to the $\mathrm{P}$ adatoms bonded with three $\mathrm{Si}$ atoms. In this configuration filled lone electron pair dangling-bond state of $\mathrm{P}$ adatoms protruding out of the surface and at negative sample bias conditions, the electrons tunnel out through this dangling bond giving brighter protrusions [11]. The protrusions due to $\mathrm{P}$ dopants which have occupied the rest-atom positions appear differently. It is believed that the bright triplets appear in STM images as shown in Figure 3(c) are due to the P dopants at rest-atom positions. The red arrows in Figure 3(c) illustrates the triplet protrusions which are responsible for $\mathrm{P}$ dopant atoms at rest-atom sites and the blue arrows shows typical $\mathrm{P}$ dopant substitutions at corner/center-adatom positions. The $\mathrm{P}$ dopant atoms at rest-atom positions also consist of the lone electron pair which is extruding out of the surface, and the electronic configuration of the three nearby adatoms get modified due to the presence of this lone electron pair. Also, the typical adatom to rest-atom charge transfer process can also be affected by the presence of this lone pair, and therefore, adatoms would not be able to charge transfer to the rest-atom positions fully giving brighter protrusions in filled state STM images. It was also observed that the brighter protrusions appear in the filled-state image for surface P-dopant atoms and the protrusions disappear from the empty-state images recorded at positive sample bias. This phenomenon is shown by arrows, where the same color arrows indicate the protrusion at the same position of filled and empty-state images, in Figure 4(a) and Figure 4(b). It further confirms that these protrusions have voltage-depended behavior and therefore the contrast in protrusions arise have an electronic origin rather than of a topological one [12].

However, the behavior of the protrusion due to the buried P-dopants behave differently as shown in Figure 4(c) and Figure 4(d). The blue arrows indicate the corresponding protrusions occur due to the subsurface P dopants. Protrusions are not visible in the filled-state STM images, but bright protrusions were observed when empty-state STM image is obtained over the same scanning area. The local Coulomb potential of buried $\mathrm{P}$ dopant ions induces a downward band bending. Thus the energetic position of the surface states become lower, and this will also reduce the valence and conduction band positions [1]. As described earlier, the electrons tunnel into empty states of the sample at positive sample bias conditions. The Higher energy states moved downwards due to the lowering effect on the local potential by the $\mathrm{P}$ ion. Therefore, the density of states which are available for incoming tunneling electrons at positive sample bias increase at the P atom giving brighter protrusions at empty-state STM images [1], [13].
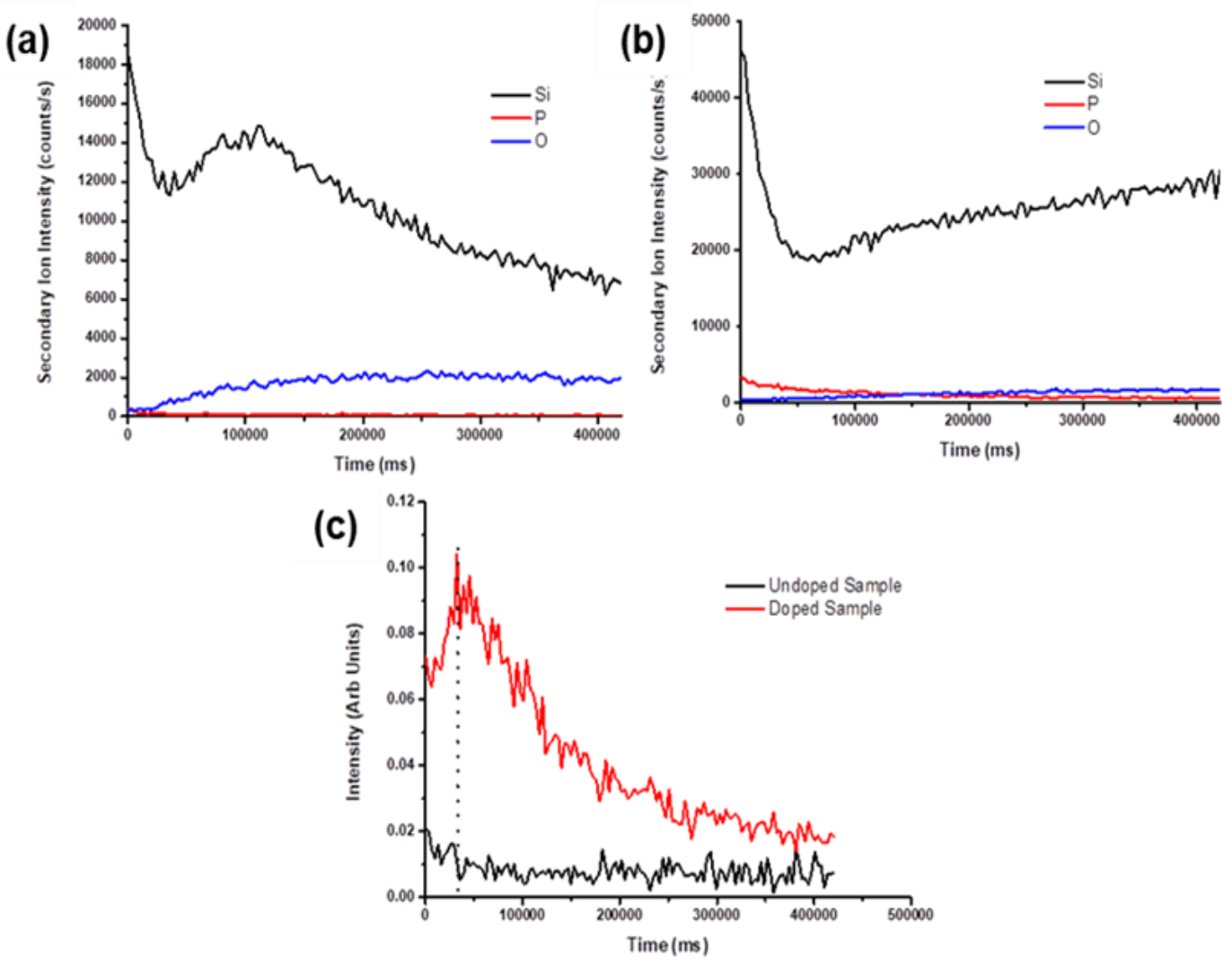

Figure 1. The raw SIMS data for obtained for (a) undoped, (b) doped Si(111) substrates and (c) plotted P/Si ratio for doped and undoped samples for comparison 


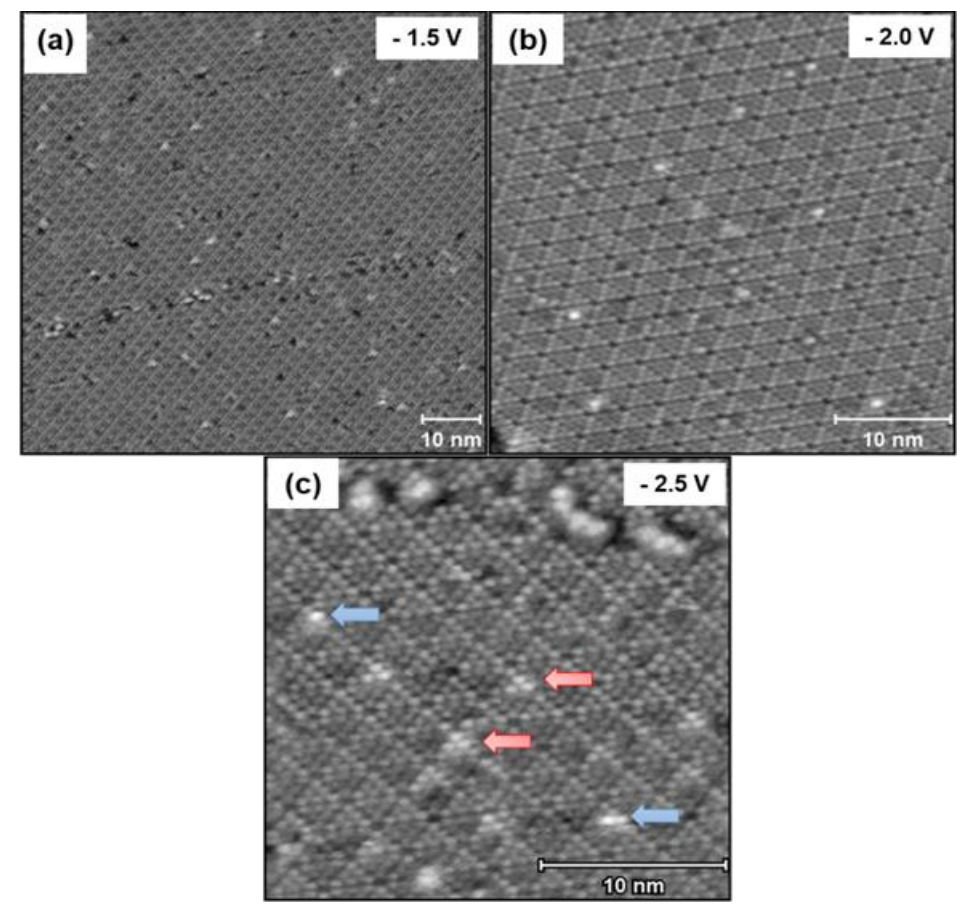

Figure 3. The STM images obtained after the $\mathrm{P}$ diffusion doping at $950^{\circ} \mathrm{C}$ for 10 minutes, (a) STM image showing large area of the surface. The bright protrusions are due to the $\mathrm{P}$ dopants, (b) Surface center-adatom and corner-adatom positions replaced by $\mathrm{P}$ dopants, (c) The bright triplet protrusions due to $\mathrm{P}$ dopants at restatom position

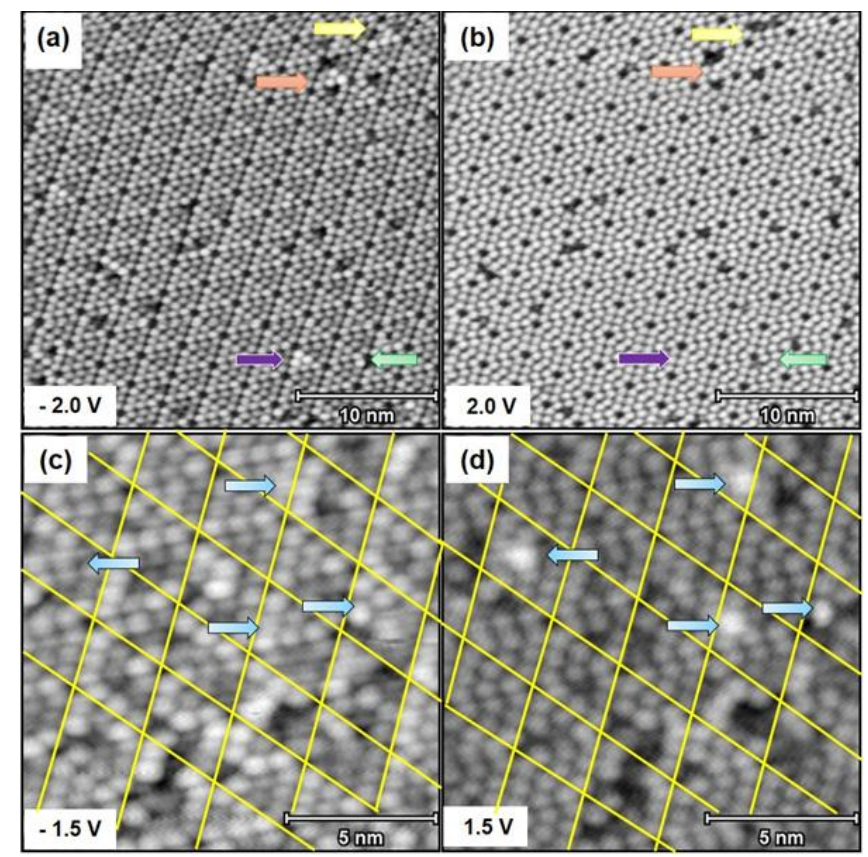

Figure 4. (a) Filled-state and (b) empty-state STM images of surface P-dopants obtained over the same scanning area, (c) Filled-state and (d) empty-state STM images showing the positions of buried P-dopants recorded over the same scanning area

The $\mathrm{Si}(111)-(7 \times 7)$ surface is again reconstructed into another stable and prevalent $\mathrm{P}$ based structure at some locations on the substrate. So-called $\mathrm{Si}(111)-(6 \sqrt{3} \times 6 \sqrt{3}) R 30^{\circ}$ reconstruction occurs at elevated 
temperatures in positions where P dopants have higher surface concentration, and a large area STM image of the reconstruction is illustrated in Figure 5(a). The surface consists of an interesting pattern of hexagonal tiles of slightly irregular shapes. These hexagonal tiles have completely tessellated the observed surface area. It was observed that these hexagonal tiles have an average diameter of $10-20 \AA$ and they have extended over the terraces to the edges of the steps hence forming irregular step edge lines. The magnified STM images shown in Figure 5(b) and Figure 5(c) demonstrate that a well-ordered (1×1) arrangement of atoms of the hexagonal domains, which are separated by straight domain walls with lower contrast. It can be concluded that the $(6 \sqrt{3} \times 6 \sqrt{3})$ domain structure is formed as the result of the extreme tensile surface stress induced by the P-Si bonding topology [14]. The $(7 \times 7)$ reconstruction get destabilized due to the high surface stress and atoms rearranged to form more stable $(6 \sqrt{3} \times 6 \sqrt{3})$ configuration with $\mathrm{P}$ dopant at the surface. The formation complete tessellation of the surface with an irregular hexagonal domain is remarkable, and this surface also provides $\mathrm{P}$ terminated configuration with dangling bonds with lone pairs. It is possible to introduced molecules with high electron affinity to interact with the surface P dopants due to this lone pairs arranged in the surface perpendicular direction.

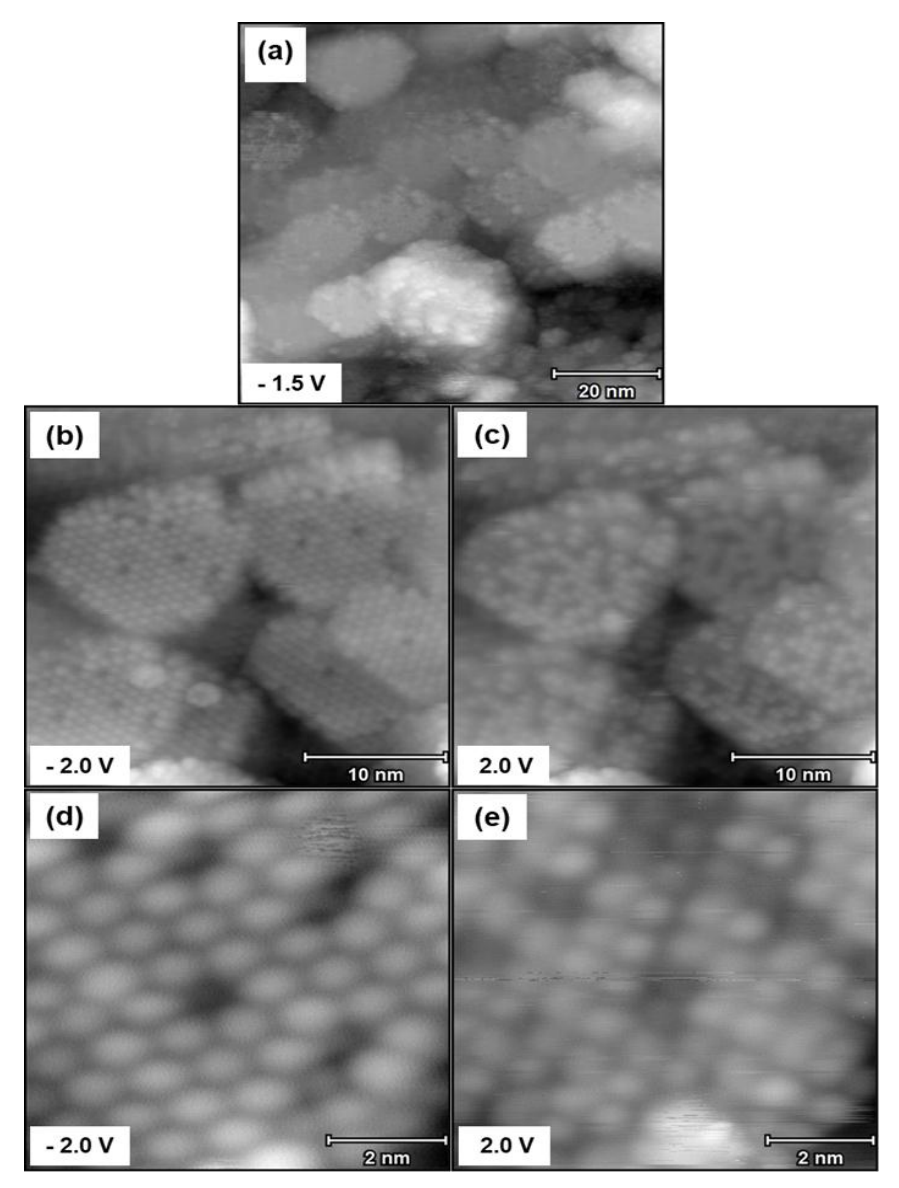

Figure 5. The STM images obtained over Si(111)- $(6 \sqrt{3} \times 6 \sqrt{3}) \mathrm{R} 30^{0}$ configuration, (a) Large area image, (b) Filled-state and (d) empty-state image obtained over the same scanning area. Magnified STM images of (d) Filled-state and (e) empty-obtained over the same scanning area

The STS analysis was carried out for all the observed surfaces to identify their electronic configurations. In typical $\mathrm{d} I / \mathrm{d} V$ spectra as a function of voltage of $\mathrm{Si}(111)-(7 \times 7)$ reconstructed surface, as shown in Figure 6(a), rest-atoms are responsible for the peak appears at $-0.8 \mathrm{~V}$. The peaks appears at $-0.35 \mathrm{~V}$ is due to the adatoms and the peak emerges at $+1.45 \mathrm{~V}$ is because of the stacking fault in $(7 \times 7)$ reconstruction [15]. After $\mathrm{P}$ doping process, newer peaks were observed at $-1.0 \mathrm{~V}$ and $-0.5 \mathrm{~V}$ which may be due to the substitution of $\mathrm{P}$ dopant atoms for $\mathrm{Si}$ atoms. Another dominant peak appears at $+0.8 \mathrm{~V}$ corresponds to the donor states located at the conduction band minimum [1]. We believe that the peak appears at $-1.0 \mathrm{~V}$ is due to tot he buried positively charged $\mathrm{P}$ atoms near rest-atom positions which can affect the filled states of 
the rest-atoms thus inducing the peak. Generally, the surface of the $(7 \times 7)$ reconstruction shows semi-metallic characteristics because of the localized adatom electrons. But, when a $\mathrm{P}$ dopant atom is get substituted in a rest-atom position the electron localization does not occur making the surface semiconducting. However, the bulk has metallic properties due to the introduction of the extra carrier electrons. This phenomenon can be clearly observed in the $I-V$ curves shown in Figure 6(b).

The electronic properties of $\operatorname{Si}(111)-(6 \sqrt{3} \times 6 \sqrt{3})$ surface was further characterized by STS analysis. The STS spectra, as shown in Figure 7(a), confirms the identity of $\mathrm{P}$ dopants of the $(1 \times 1)$ features giving a surface-state band gap of $1.7 \mathrm{eV}$ [11]. This band gap value is comparable to the surface-state band gap of $1.5 \mathrm{eV}$ observed in $\mathrm{Si}(111)-(1 \times 1)-\mathrm{As}$ surface previously [16]. Therefore, it can be concluded that a Pterminated $(1 \times 1)$ surface has formed due the excess $\mathrm{P}$ atoms which have converged into a small area.
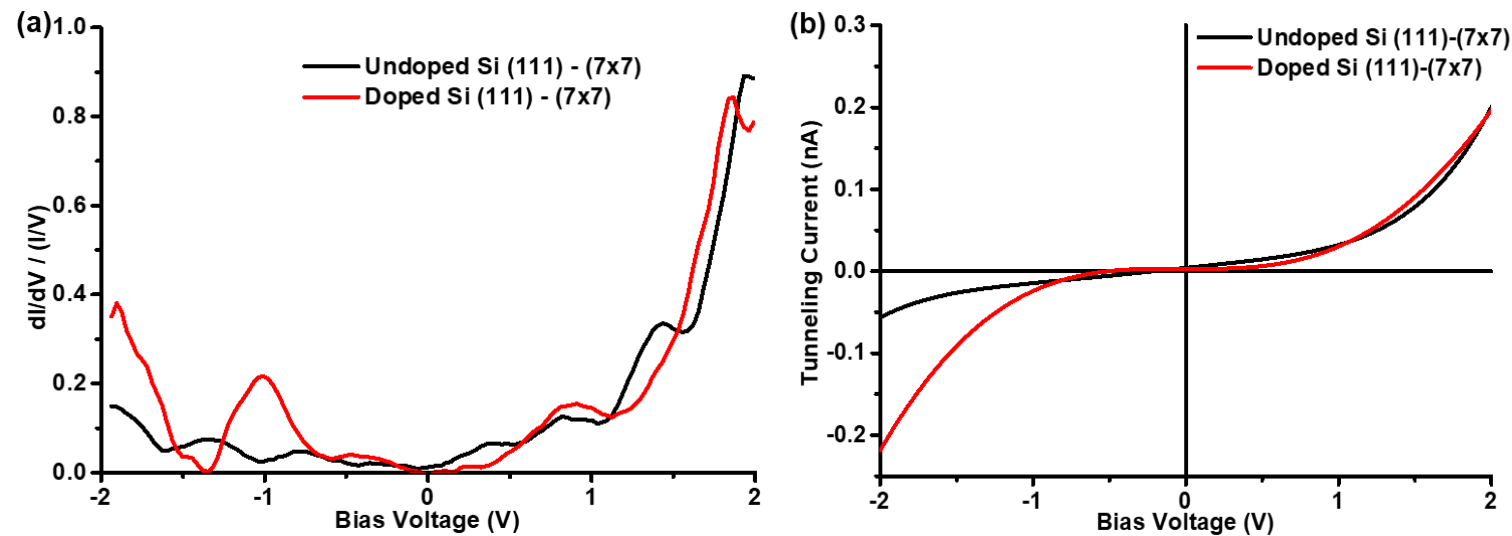

Figure 6. The STS analysis of P-doped and normal Si (111) - (7×7) reconstructions, (a) The first derivative of tunneling current against sample bias and (b) I-V curves for the two samples
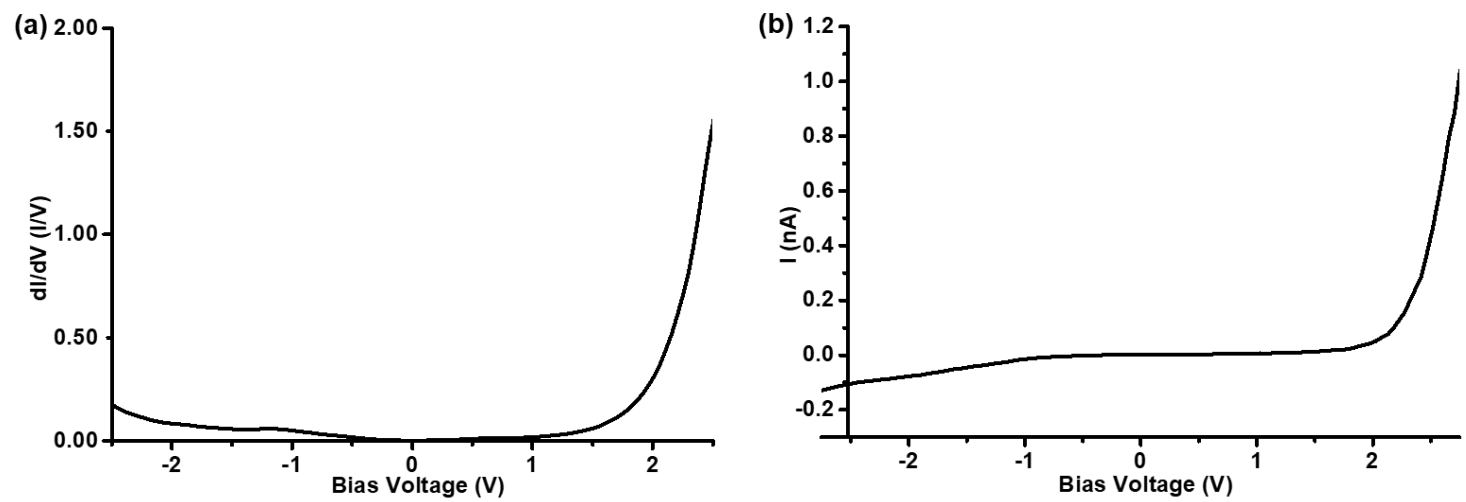

Figure 7. The STS spectra of $\operatorname{Si}(111)-(6 \sqrt{3} \times 6 \sqrt{ } 3) \mathrm{R} 30^{0}$ reconstruction, (a) First derivative of tunneling current against sample bias and (b) corresponding I-V curve

\section{CONCLUSION}

The Si(111) surface was further modified by introducing excess $\mathrm{P}$ dopants to the substrate. The thermal diffusion process was used as the P doping method. The four-probe-point measurements and SIMS measurements confirm the high $\mathrm{P}$ doping after the thermal diffusion process. The $\mathrm{Si}(111)$ substrates were cleaned by wet HF etching and then introduced into the STM chambers followed by several cycles of the flash heating process. Random bright protrusions were observed in filled-state STM images, and it was confirmed that these bright spots are due to surface $\mathrm{P}$ dopant atoms substituted to adatom positions. The $\mathrm{P}$ dopants have lone pair dangling bonds protruding out of the substrate surface which responsible for giving bright protrusions at filled-state STM images. These bright protrusions were not observed in corresponding empty-state STM images confirming that these protrusions have a topological origin. It was also observed that $\mathrm{P}$ dopant atoms placed at rest-atom positions give bright triplet like spots. When a $\mathrm{P}$ dopant occupies a 
rest-atom position, it disrupts the adatom to rest-atom charge transfer process. Therefore, adatoms nearby would have higher electron density than other adatoms giving brighter triplet like protrusions.

However, subsurface P dopants act differently than the surface P dopants. Bright protrusions were observed in the empty-state STM images but not observed in the corresponding filled-state STM images. At subsurface level, the positive Coulomb potential exerted by $\mathrm{P}$ dopant ion would induce a downward band bending thus increasing the density of empty states for electrons to tunnel into giving bright protrusions. The STS study reveals several peaks due to the dopant $\mathrm{P}$ atoms. Also, semi-metallic $(7 \times 7)$ reconstruction has become semiconducting upon $\mathrm{P}$ doing. However, the bulk shows metallic properties due to high $\mathrm{P}$ doping.

P-terminated $\mathrm{Si}(111)-(6 \sqrt{3} \times 6 \sqrt{3})$ reconstruction which has hexagonal tiles of slightly irregular shapes was observed at various positions where higher $\mathrm{P}$ dopant concentrations are available. The $(7 \times 7)$ reconstruction get destabilized due to the high surface stress and atoms rearranged to form more stable $(6 \sqrt{3} \times 6 \sqrt{3})$ configuration with $P$ dopant at the surface. This surface is interesting since the availability of lone pair dangling bonds to attach various molecules. The availability of surface $\mathrm{P}$ dopant atoms was also confirmed from the surface band gap of $1.7 \mathrm{eV}$ in the STS spectra.

\section{ACKNOWLEDGEMENTS}

Part of this research was supported by JSPS KAKENHI, Grant Number 17 K05055.

\section{REFERENCES}

[1] T. Trappmann, C. Sürgers, and H. v. Löhneysen, "Observation of P donors on the Si(111) surface by scanning tunneling microscopy," Europhysics Letters (EPL), vol. 38, pp. 177-182, 1997.

[2] F. F. Y. Wang, "Introduction to Solid State Electronics", Elsevier Science, 2012.

[3] A. Many and Grover, "Semiconductor surfaces", Elsevier, 1965.

[4] Y. P. Zhang and G. Q. Xu, "Self-Assembled Molecular Corrals Formed on Si(111)-(7 x 7) Surface via Covalent Bond," Journal of Physical Chemistry C, vol. 114, pp. 16625-16629, 2010.

[5] M. Shimomura, A. Iwanabe, and T. Kiyose, "Dynamic Observation of Confined Molecules in Self-Assembled Molecular Corrals," The Journal of Physical Chemistry C, vol. 118, pp. 27465-27469, 2014.

[6] M. Shimomura, N. Sanada, Y. Fukuda, and P. J. Møller, "Highly site-selective adsorption of trimethylphosphine on a Si(111)-(7 × 7) surface studied by a scanning tunneling microscope $(\mathrm{STM}), "$ Surface Science, vol. 341, pp. L1061-L1064, 1995.

[7] H. D. Siriwardena and M. Shimomura, "Effect of Tunneling Electron Injection on the Dynamic Motion of Confined Molecules in Self-Assembled Molecular Corrals," The Journal of Physical Chemistry C, vol. 121, pp. 4980-4988, 2017.

[8] W. Mönch, "Semiconductor Surfaces and Interfaces", Springer Berlin Heidelberg, 2013.

[9] L. W. Turner, "Electronics Engineer's Reference Book", Elsevier Science, 2013.

[10] D. K. Schroder, "Semiconductor Material and Device Characterization", Wiley, 2006.

[11] L. Vitali, M. G. Ramsey, and F. P. Netzer, "Substitutional geometry and strain effects in overlayers of phosphorus on Si(111)," Physical Review B, vol. 57, pp. 15376-15384, 1998.

[12] T. Trappmann, C. Surgers, and H. von Lohneysen, "Investigation of the (111) surface of P-doped Si by scanning tunneling microscopy," Applied Physics a-Materials Science \& Processing, vol. 68, pp. 167-172, 1999.

[13] S. Kurokawa, T. Takei, and A. Sakai, "A Search for Subsurface Dopants on Hydrogen-Terminated Si(111) Surfaces," Japanese Journal of Applied Physics, vol. 42, pp. 4655-4658, 2003.

[14] E. Kaxiras, "Atomic-structure of surfactant monolayers and its role in epitaxial-growth," Materials Science and Engineering B-Solid State Materials for Advanced Technology, vol. 30, pp. 175-186, 1995.

[15] T. Berghaus, A. Brodde, H. Neddermeyer, and S. Tosch, "On the interpretation of current images in scanning tunneling spectroscopy of SI(111) -7×7," Journal of Vacuum Science \& Technology a-Vacuum Surfaces and Films, vol. 6, pp. 483-487, Mar-Apr 1988.

[16] R. I. G. Uhrberg, R. D. Bringans, M. A. Olmstead, R. Z. Bachrach, and J. E. Northrup, "Electronic structure, atomic structure, and the passivated nature of the arsenic-terminated Si(111) surface," Physical Review B, vol. 35, pp. 3945-3951, 03/15/ 1987. 


\section{BIOGRAPHIES OF AUTHORS}
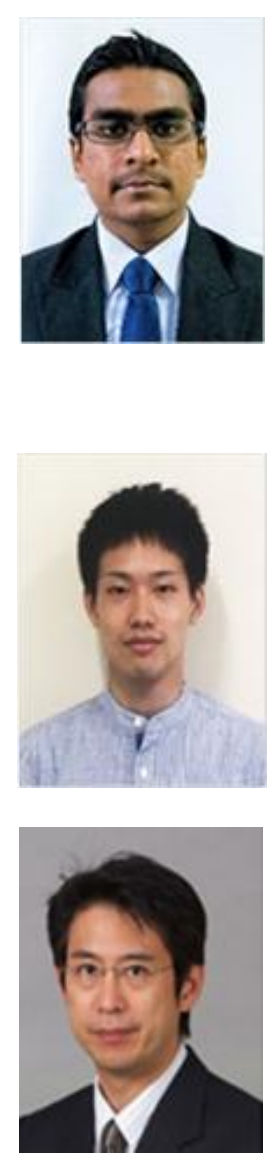

Hirulak D. Siriwardena obtained his bachelor's degree from the Faculty of Science, the University of Peradeniya with honors in 2011. After completing his master's degree in nanoscience and nanotechnology in 2013 at the postgraduate institute of science, University of Peradeniya, he joined surface and materials science research group of Prof. Masaru Shimomura, Shizuoka University in 2014 as a doctoral research student. His research interests are focus on studying the surface chemistry of molecules and different substrates for the fabrication of single molecular type electronic devices using scanning tunneling microscope. Additionally, he is also collaboratively developing graphene oxide $/ \mathrm{TiO}_{2}$ composite for potential gas sensor applications and $\mathrm{TiO}_{2}$ nanotubes based dye-sensitized solar cells with high open circuit voltages. He is a graduate student member of Japanese surface science society and Japanese society of applied physics.

Toru Yamashita completed his bachelor's degree in 2016 at Fuculty of Engineering, Shizuoka University. He is currently completing his master's degree at the Graduate School of Integrated Science and Technology, Shizuoka University. He is currently studying atomic-level and molecular-level effects aimed at developing future generations of electronics in semiconductor nano-devices and nano-structures.

Masaru Shimomura is a professor in the Department of Electronics and Materials Science, Faculty of Engineering, Shizuoka University. He completed his bachelor's degree in 1992 from Faculty of Engineering, Shizuoka University and obtained his master's degree from Graduate School of Engineering, Shizuoka University in 1994. Then, he completed the Ph. D. degree in Engineering from Division of Electronic Science, Shizuoka University in 1997. After Ph. D., he started academic job as a research associate at Research Institute of Scientific Measurements, Tohoku University. In 2002, he returned to Shizuoka University as a research associate. Then, promoted an associate professor in 2008, and a professor in 2015. He is an expert in surface chemistry and sophisticated surface analytical systems such as XPS and STM. His research group studies the structure of surfaces and interfaces of materials for future electronic devices in atomic or molecular scale. Controlling of the surface structure of nano particles for dyesensitized solar cells or gas sensors is another research topic his group is currently studying. 